\section{Microbiological assessment of freshly-shot wild boars meat in Lazio Region, Viterbo territory: a preliminary study}

\author{
Eda Maria Flores Rodas, ${ }^{1}$ \\ Tatiana Bogdanova, ${ }^{1}$ Teresa Bossù, ${ }^{1}$ \\ Sabrina Pecchi, ${ }^{1}$ Francesco Tomassetti, ${ }^{1}$ \\ Paola De Santis, ${ }^{1}$ Rita Tolli,' \\ Roberto Condoleo, ${ }^{1}$ Sara Greco, ${ }^{1}$ \\ Alberto Brozzi,' Stefano Bilei,' \\ Giuseppe Micarelli, ${ }^{2}$ Enrica Martini, \\ Massimo Palazzetti \\ 1'Istituto Zooprofilattico Sperimentale \\ delle Regioni Lazio e Toscana, Roma; \\ ${ }^{2}$ Azienda Sanitaria Locale 9 - Sezione \\ di Viterbo, Italy
}

\section{Abstract}

During the wild boars hunting season (2012-2013), 6508 adults were shot in Lazio Region, Viterbo territory. Out of the 6508, 393 subjects were sampled to be tested for the detection of Salmonella spp., Yersinia enterocolitica, E. coli VTEC and Trichinella spp. For this purpose, diaphragm and skeletal muscles samples were collected after the shooting, and tested at the Istituto Zooprofilattico Sperimentale Lazio and Toscana laboratories, Rome, Italy. Samples were tested respectively by polymerase chain reaction, enzyme linked immunofluorescent assay, microbiological and parasitological assays. The positivity percentages are 3.6\% for Salmonella spp., 14.8\% (out of 230 samples tested) for Yersinia enterocolitica, $4.6 \%$ for E.coli VTEC (vtx 1 and 2 genes). Each positive sample for $v t x 1$ and 2 was tested to isolate Verotoxigenic E.coli strains; none of them was positive for the same virulence genes. All diaphragm samples were negative to the trichinoscopic test. Every bacterial strain was identified serologically by seroagglutination test.

\section{Introduzione}

La catena alimentare rappresenta una delle principali vie di trasmissione di zoonosi (Milnes et al., 2008). Durante gli ultimi anni, la popolazione di suidi selvatici è aumentata notevolmente in tutta Europa, probabilmente in relazione all'innalzamento delle temperature ed all'aumento delle fonti di alimento (Geisser e Reyer, 2005). L'aumento della densità della popolazione di cinghiali, unita allo sviluppo di allevamenti semibradi di suini dome- stici, può rappresentare un fattore di rischio per la trasmissione di microrganismi e parassiti tra le due popolazioni (Gortázar et al., 2006). Quindi, i cinghiali possono rappresentare un reservoir di agenti patogeni per i suini domestici (de Deus et al., 2008).

Salmonella spp., Yersinia enterocolitica, Verotoxigenic E. coli (VTEC) e Trichinella spp. sono patogeni di origine alimentare sono diffusi nei suini domestici e nel loro ambiente e la trasmissione tra animale ed animale avviene principalmente per via oro-fecale (Butzler, 2004). L'uomo si infetta attraverso il consumo di acqua od alimenti contaminati 0 attraverso lo stretto contatto con animali infetti ma asintomatici. In contrasto con l'abbondante letteratura disponibile sulla presenza dei suddetti patogeni zoonosici nell'uomo e nei suini domestici, si conosce molto poco sulla presenza di questi patogeni nelle popolazioni di suidi selvatici. Per questo motivo ed anche per l'aumento del consumo pro capite di carne di cinghiale, cotta 0 cruda stagionata, la maggiore conoscenza della circolazione di patogeni zoonosici a trasmissione alimentare sta diventando un argomento di salute pubblica sempre più importante (Wacheck et al., 2010).

Lo scopo del presente studio è stato quello di valutare il grado di diffusione di patogeni a trasmissione alimentare nella popolazione di cinghiali durante gli abbattimenti programmati nel periodo 2012-2013 nel comprensorio di Viterbo.

\section{Materiali e metodi}

\section{Raccolta campioni}

I campioni sono stati prelevati durante le battute di caccia effettuate nel comprensorio di Viterbo nei periodi dal 6/1/2012 al $30 / 01 / 2012$, dal 12/11/2012 al 27/11/2012 e dal 5/01/2013 al 17/03/2013, sia come abbattimenti programmati che come abbattimenti per il controllo demografico di popolazione. I soggetti abbattuti e campionati sono stati complessivamente 393, di cui 170 (43,3\%) maschi, 215 $(54,7 \%)$ femmine e $8(2,0 \%)$ soggetti per i quali il sesso non è stato dichiarato nel verbale di prelevamento, dai quali sono stati prelevati campioni di muscolo (diaframma e muscolo scheletrico, prevalentemente della coscia) per le ricerche batteriologiche (Salmonella spp, $Y$. enterocolitica ed $E$. coli VTEC) e parassitologiche (Trichinella spp.). Tutti gli esami sono stati effettuati pressi i Laboratori dell'Istituto Zooprofilattico Sperimentale delle Regioni Lazio e Toscana, Roma.

\section{Prove di laboratorio}

\section{Analisi microbiologiche}

Come metodo di screening per la ricerca di
Correspondence: Eda Maria Flores Rodas, Istituto Zooprofilattico Sperimentale delle Regioni Lazio e Toscana, via Appia Nuova 1411, 00178 Roma, Italy.

Tel. +39.06.79099340 - Fax: +39.06.79099330.

E-mail: eda.flores@izslt.it

Key words: Wild boars, Foodborne pathogens, Salmonella spp., Yersinia enterocolitica, Escherichia coli.

Conflict of interests: the authors declare no potential conflict of interests.

Received for publication: 13 May 2013

Revision received: 9 September 2013.

Accepted for publication: 25 September 2013

This work is licensed under a Creative Commons Attribution 3.0 License (by-nc 3.0).

CCopyright E.M. Flores Rodas et al., 2014 Licensee PAGEPress, Italy

Italian Journal of Food Safety 2014; 3:1711 doi:10.4081/ijfs.2014.1711

Salmonella spp. è stata utilizzata la procedura VIDAS-SLM. Il metodo VIDAS consiste essenzialmente in un'analisi immuno-enzimatica a fluorescenza effettuata mediante lo strumento VIDAS $^{\circledR}$ bioMérieux (Marcy l'Etoile, Francia). Nel caso di positività dei brodi, è stata eseguita anche la prove colturale secondo la ISO 6579:2002. Le colonie sospette Salmonella spp sono state confermate con VITEK $^{\circledR} 2$ Compact, e successivamente sottoposte a sierotipizzazione. Per la ricerca di Yersinia enterocolitica è stata utilizzata la metodica ISO 10273:2003. Le colonie sospette sono state confermate con VITEK2 ${ }^{\circledR}$ COMPACT BioMérieux e quindi saggiate in real time polymerase chain reaction (PCR) per il rilevamento del gene ail che rappresenta un carattere di virulenza. Le stesse colonie sono quindi state sottoposte a sierotipizzazione. Per la ricerca di E.coli VTEC è stato utilizzato il protocollo descritto nella ISO/TS 13136. I campioni risultati positivi per la presenza di geni codificanti per i fattori di virulenza (eae, $v t x 1$, $v t x 2$ ) allo screening in PCR sono stati seminati su appositi terreni in piastra (S-MAC, CT-SMAC e chromID ${ }^{\circledR}$ BioMérieux) previa immunoseparazione, per 0:157 ed 0:26, oppure omettendo questo passaggio per tutti gli altri sierogruppi ricercati, a causa della scarsa efficacia dei kit presenti in commercio. Le colonie isolate sono state nuovamente saggiate mediante PCR per la presenza dei geni codificanti per i fattori di virulenza (eae, vtx1, vtx2) e per la definizione del sierogruppo, e quindi sottoposte a sieroagglutinazione rapida su vetrino.

\section{Sierotipizzazione}

Tutti i ceppi di Salmonella spp isolati sono 
stati sottoposti a sieroagglutinazione rapida su vetrino. I sieri utilizzati sono Polivalenti $0, \mathrm{H} \mathrm{e}$ Vi [Statens Serum Institut (SSI), Copenhagen, Danimarca].

Tutti i ceppi di Yersinia enterocolitica isolati sono stati sottoposti a sieroagglutinazione rapida. I sieri utilizzati sono Polyvalent 01-02 e Group 03, 05,08 ed 09 (Denka Seiken Co. Ltd., Tokyo, Giappone).

Tutti i ceppi di $E$. coli risultati positivi per almeno 1 fattore di patogenicità $v t x 1$ o/e $v t x 2$ sono stati esaminati con il metodo PCR per l'identificazione del sierogruppo. Tutti i ceppi di E.coli sono stati sottoposti a sieroagglutinazione rapida su vetrino. I sieri utilizzati sono Pool E.coli (SSI) e monovalenti 026, 0103 , 0104, 045, 0111, 0157, 0121.

\section{Ricerca di Trichinella spp.}

La ricerca di Trichinella spp. è stata eseguita secondo la metodica descritta dal Regolamento 2075/2005/CE.

\section{Risultati}

Le indagini eseguite hanno dato i seguenti risultati. Salmonella spp. è stata rilevata nel $3,6 \%$; i 7 ceppi saggiati sono stati identificati come: 1 ceppo S. Kottbus, 2 ceppi S. Manhattan e 4 ceppi S.enterica subsp. diarizonae IIIb.

E. coli VTEC nel $4,6 \%$ dei soggetti campionati (vtx 1 e 2 rilevati insieme in 12 campioni, e solo il gene $v t x 1$ in 6 campioni. I ceppi isolati da 14 dei 18 campioni saggiati, sono risultati appartenenti ai seguenti sierogruppi: 6 campioni 0145,5 campioni 0157,1 campione 0104, 1 campione 026 ed 1 campione 0103.

Yersina enterocolitica è stata rilevata nel $14,8 \%$ su un totale di 230 campioni sottoposti a tale prova). I 34 ceppi isolati sono stati identificati come 3 appartenenti al sierogruppo 08, 1 al sierogruppo 03, 2 al sierogruppo 05 e 28 ceppi non agglutinanti con i sieri suddetti.

La ricerca di Trichinella spp. ha dato esito negativo in tutti i 393 campioni sottoposti a saggio.

\section{Discussione e Conclusioni}

La qualità microbiologica della carne di selvaggina abbattuta dipende da diversi fattori: i) i tipi di microrganismi presenti nel tratto gastroenterico, nei muscoli ed in altre sedi di latenza degli animali; ii) circostanze e modalità di abbattimento; iii) condizioni di sezionamento della carcassa. La microflora che si svilupperà durante la conservazione delle carni sarà correlata alle condizioni di conservazione ed alle caratteristiche biochimiche intrinseche della carne stessa. Sembra esserci una differenza significativa di contaminazione batterica anche a seconda delle zone colpite in fase di caccia. Infatti, contaminazioni maggiori sono state rilevate in quei soggetti uccisi con colpi all'addome piuttosto che in altre parti del corpo, probabilmente per danno intestinale e fuoriuscita di feci (Avagnina et al., 2009). Altro interessante dato scaturito è la differenza nella percentuale di soggetti infetti tra femmine, $29,3 \%$ del totale delle femmine, e maschi, $22,4 \%$ del totale dei maschi. Tale differenza è stata già riportata da altri Autori (Wacheck et al., 2010) e sembra essere legata al diverso comportamento sociale tra maschi e femmine. Queste ultime, infatti, vivono prevalentemente in un contesto sociale di comunità con altre femmine e cuccioli, mentre i maschi sono prevalentemente solitari. Relativamente al rapporto tra peso dei soggetti abbattuti, parametro utile al fine di classificare le differenti fasce di età, e percentuale di soggetti infetti, dai dati a nostra disposizione risulta che nei soggetti di peso fino a $50 \mathrm{~kg}$ la percentuale di infetti è del $37,2 \%$, mentre in quelli di peso superiore ai 50 $\mathrm{kg}$ la percentuale si riduce al $29,9 \%$. Ciò è riferito in bibliografia (Wacheck et al., 2010) e sembra essere legato al comportamento sociale dei soggetti più giovani che vivono in comunità rispetto agli adulti spesso solitari (Kranker et al., 2003).

È ormai noto che la fauna selvatica rappresenta una fonte di infezioni di vario tipo per gli animali domestici. I cinghiali, in particolare, sono spesso portatori di agenti di zoonosi a trasmissione alimentare a livello tonsillare, ma non solo (Milnes et al., 2008). I portatori rappresentano, quindi, una fonte di contaminazione delle carcasse. Questo è un aspetto che anche i cacciatori devono tenere ben presente, trovandosi spesso a manipolare carcasse in scarse condizioni igieniche. Ciò può costituire un problema anche a livello domestico, non tanto per il consumo diretto della carne di cinghiale, generalmente ben cotta, ma per il rischio di contaminazioni crociate con altri alimenti che poi non subiranno trattamenti termici o di sanificazione (Milnes et al., 2008). In generale, il consumo di carne di cinghiale poco cotta è comunque da sconsigliare.

Infine, da non trascurare è anche il possibile contatto tra i cinghiali vivi ed i suini domestici allevati all'esterno e la possibile trasmissione di questi patogeni dai selvatici ai domestici (Wacheck et al., 2010).

\section{Bibliografia}

Avagnina A, Ferroglio E, Grassi MA, Civera T, 2009. Investigazion about microbiological quality of freshly shot game from alpine ungulates. Disponibile al sito: http://www. izsler.it/izs_bs/allegati/718/09SIEF.pdf

Butzler JP, 2004. Campylobacter, from obscurity to celebrity. Clin Microbiol Infec 10:86876.

Deus de N, Peralta B, Pina S, Allepiz A, Mateu E, Vidal D, Ruiz-Fons F, Martìn M, Gortàzar C, Segalés J, 2008. Epidemiological study of Hepatitis E virus infection in European wild boars (Sus scrofa) in Spain. Vet Microbiol 129:163-70.

Geisser H, Reyer HU, 2005. The influence of food and temperature on population denisty of wild boar Sus scrofa in the Thurgau (Switzerland). J Zool 267:89-96.

Gortázar C, Acevedo P, Ruiz-Fons F, Vincente J, 2006. Disease risks and overabundance of game species. Eur J Wildlife Res 52:81-7.

Kranker S, Alban L, Boes J, Dahl J, 2003. Longitudinal study of Salmonella enterica serotype Typhimurium infection in three Danish farrow-to-finish swine herds. J Clin Microbiol 41:2282-8.

Milnes AS, Steuart I, Clifton-Hadley FA, Davies RH, Newell DG, Sayers AR, Cheasty T, Cassar C, Ridley A, Cook AJC, Evans SJ, Teale CJ, Smith RP, McNally A, Toszeghy M, Futter R, Kay A, Paiba GA, 2008. Intestinal carriage of verocytotoxigenic Escherichia coli 0157, Salmonella, thermophilic Campylobacter and Yersinia enterocolitica, in cattle, sheep and pigs at slaughter in Great Britain during 2003. Epidemiol Infect 136:739-51.

Wacheck S, Fredriksson-Ahomaa M, Konig M, Stolle A, Stephan R, 2010. Wilde Boars as an important reservoir for foodborne pathogens. Foodborne Pathog Dis 7:30712. 